International Journal of Difference Equations (IJDE).

ISSN 0973-6069 Volume 14, Number 2 (2019), pp. 195-206

(C) Research India Publications

https://dx.doi.org/10.37622/IJDE/14.2.2019.195-206

\title{
Sinc Method for Two-Dimensional Volterra Integral Equations of First and Second Kinds
}

\author{
Yousef Al-jarrah", Taqi A. M. Shatnawi ${ }^{2}$ \\ ${ }^{1}$ Mathematics Department, Tafila Technical University, Tafila, Jordan. \\ ${ }^{2}$ Department of Mathematics, The Hashemite University, Zarqa.
}

\begin{abstract}
A numerical technique based on the Sinc collocation method is presented for the solution of two-dimensional Volterra integral equations of first and second kinds. The Sinc function properties are provided and the global convergence analysis is obtained to guarantee the efficiently of our method. Finally, we apply the method for some numerical examples to ensure the solution convergent. Comparing with other methods, Sinc method is more efficient and easy to use.
\end{abstract}

Keywords: Volterra, Two-Dimensional, Sinc Approximation.

\section{INTRODUCTION}

Several problems in engineering and physics are formulated as an integral equation. Two-dimensional Volterra integral equations provide an important tool for modeling different problems, these equations appears in electromagnetic and electrodynamic, elasticity and dynamic contact, heat and mass transfer, fluid mechanic, acoustic, chemical and electrochemical process, molecular physics, population, medicine and in many other fields [1].

So many researchers encouraged to provide a numerical solution for integral equations, where some of integral equations have no analytical solutions or it is difficult to finding their analytical solutions.

We consider the two-dimensional linear Volterra integral equation of the first and second kind respectively of the form

$$
\begin{aligned}
& g(x, y)=\int_{a}^{x} \int_{a}^{y} k(x, y, s, t) f(s, t) d s d t \\
& f(x, y)=g(x, y)+\int_{a}^{x} \int_{a}^{y} k(x, y, s, t) f(s, t) d s d t
\end{aligned}
$$


Here, $f(x, y)$ is the unknown function, we assume that the functions $f, g$ are sufficiently smooth $\operatorname{for}(x, y) \in[a, b]^{2} \times[a, b]^{2}$, and $k(x, y, s, t)$ is continuous on $[a, b]^{2} \times[a, b]^{2}$.

The numerical solution of integral equation has been growing rapidly. But Comparing with the numerical analysis of one-dimensional integral equations, the analysis of computational methods for two-dimensional integral equations has started recently and has difficulty to solve. Numerous articles have been suggested numerical solution for equations (1.1) , (1.2) and their nonlinear form. Firstly, a class of explicit RungeKutta method is used to solve the nonlinear two-dimensionl Volterra integral equations, but without analyzing their convergence [2], Bivariate cubic spline was used by Singh to solve the equations (1.1) and (1.2). Secondly, Brunner and Kuathen provided a numerical solution for the two-dimensional Volterra integral equations by collocation and iterated collocation methods, where the global convergence and superconvergent were provided for the methods [3]. In additions, the same method is used to solve the nonlinear part and the accuracy of the solution was improved by using Richardson' extrapolation [3]. Moreover, the two-dimensional piecewise constant block-plus functions was used by [4] to the nonlinear part of (1.1). Moreover, Garlerkin and iterated Garelkin were suggested in [5], and Homotopy perturbation and differential transform [6] wrere used to solve equations(1.1), (1.2). Another different methods were suggested a numerical solution of equations (1.1) (1.2) as in [7], [8], [9] and [10]. Finally, Haar wavelet basis function had shown an efficient method for solving integral equations because of properties of wavelet functions [11], where different wavelet basis function could be used to obtain a numerical solution, like Coiflets, Duabaches and Shannon wavelets, where the Coiflets were used for solving different kinds of one-dimensional Volterra and Fredholm integral equations and two-dimensional Fredholm integral equations [12], [13] and [14].

In this paper, the Sinc function is used to obtain the numerical solution for equations (1.1) and (1.2). where the Sinc functions shown an efficient method for solving integral equations. The Sinc method was used to solve one-dimensional Fredholm and Volterrra integral equations [15], [16] . In addition, singular integral equations, integro-diiferntial equations and Fredholm-Volterra integral equations.

\section{SINC FUNCTION}

In this section, we will give a brief introduction of the Sinc function and it's properties, in addition to some definitions and theorems that are required for function approximation. The Sinc function is defined in the real line as follows

$$
\operatorname{sinc}(x)=\left\{\begin{array}{ll}
\frac{\sin (x)}{x} & x \neq 0 \\
1 & x=0
\end{array},\right.
$$


and the normalized Sinc function has the form

$$
\operatorname{Sinc}(x)= \begin{cases}\frac{\sin (\pi x)}{\pi x} & x \neq 0 \\ 1 & x=0\end{cases}
$$

This function is defined by Borel and Whittaker. For any $h>0$ the Sinc function (1.3) is translated with spaced nodes $i h$ and scaled by $h$ as follows;

$$
S(j, h)(x)=\operatorname{Sinc}\left(\frac{x-j h}{h}\right), j=0, \pm 1, \pm 2, \ldots .
$$

Definition 1. Let $H^{1}\left(\mathrm{D}_{E}\right)$ denote the family of all analytic functions in the infinite strip

$$
D_{E}=\{z=u+i v ;|\operatorname{Im}(z)|=|v|<d, d \leq \pi / 2\} .
$$

For a given function $f(x),-\infty<x<\infty$, the Sinc function interpolation is defined by the Sinc basis functions $S(j, h)(x)$

$$
\begin{aligned}
\mathrm{D}_{d}^{2}= & \left.\left\{z \in C ;\left|\arg \left(\frac{z-a}{b-z}\right)\right|<d\right\},-\infty<a<b<\infty\right\} \\
& P^{N}(f)(x)=\sum_{k=-\infty}^{\infty} f(k h) S(k, h)(x)
\end{aligned}
$$

The function approximation (1.5) is known as Whittaker expansion. In fact (1.5) gives the function approximation on the whole real line, and to have an approximation for function which is defined on closed interval $[a, b]$, we consider a conformal map $\varphi(x)$ defines the Sinc function over a closed interval $[a, b]$ as follows.

$$
\varphi(x)=\ln \frac{x-a}{b-x}
$$

which maps the eye-shaped region $\left\{z=x+i y ;\left|\arg \left(\frac{z-a}{b-z}\right)\right|<d<\frac{\pi}{2}\right\}$ onto $D_{E}$, and the $\varphi(x)$ is a one-to-one function on the interval $(a, b)$ onto the real line. Therefore, the basis functions on the interval $[a, b]$ are given by the composition

$$
S(j, h)(x) \circ \varphi(x)=\operatorname{Sin}\left(\frac{\varphi(x)-j h}{h}\right) .
$$


The Sinc collocation points $x_{k}$ are defined for $h>0$ by

$$
x_{k}=\varphi^{-1}(k h)=\frac{a+b e^{k h}}{1+e^{k h}}, k=0, \pm 1, \pm 2, \ldots
$$

Theorem 1. Let $f(x) \in L_{\alpha}\left(D_{E}\right)$. Then there exist a positive constants $c_{0}$ and $r_{0}$ independent of $N$, such that

$$
\sup _{x \in \Gamma}\left|f(z)-\sum_{k=-N}^{N} f\left(z_{k}\right) S(k, h) \circ \varphi(z)\right|<c_{0} \exp (-(\pi d \alpha N))^{\frac{1}{2}}
$$

where $N$ is appositive integer and $h=\sqrt{\frac{\pi d}{\alpha N}}$.

For a continuous function $f(x, y)$ is on the rectangle $[a, b]^{2}$, then the Sinc interpolation is defined as

$$
P_{N}(f)(x, y)=\sum_{k=-N}^{N} \sum_{k^{\prime}=-N}^{N} f\left(x_{k}, \mathrm{y}_{k^{\prime}}\right) S(k, h) \circ \varphi(x) S\left(k^{\prime}, h\right) \circ \varphi(y)
$$

Where $x_{k}$ and $y_{k^{\prime}} k, k^{\prime}=-N, \ldots, N$ are the Sinc collocation points defined in (1.7) and $h=\left(\frac{\pi d}{\alpha N}\right)^{\frac{1}{2}}$. Theorem 2: For a given constants $\alpha, d$ and integer $N, h=\left(\frac{\pi d}{\alpha N}\right)^{\frac{1}{2}}$, and $P_{N}(f)(x)$ is the Sinc interpolation for the function $f(x, y)(1.9)$. Then

$$
\sup _{(x, y) \in[a, b]^{2}}\left|f(x, y)-P_{N}(f)(x, y)\right| \leq\left(c_{1}+c_{2} \log N\right) N^{\frac{1}{2}} \exp \left(-(\alpha d \pi N)^{\frac{1}{2}}\right)
$$

Where, $c_{1}$ and $c_{2}$ are constants independent of $N$.

The proof exists in [17].

\section{SINC-METHOD FOR TWO-DIMENSIONAL VOLTERRA INTEGRAL EQUATION}

In this section, we will use the Sinc basis functions for approximating the unknown function $f(x, y)$ of the integral equations (1.1) and (1.2). Firstly, inserting (1.9) into equation (1.1), then we have

$$
g(x, y)=\int_{a}^{x} \int_{a}^{y} k(x, y, s, t) \sum_{k=-N}^{N} \sum_{k^{\prime}=-N}^{N} f_{k, k^{\prime}} S_{k}(\varphi(s)) S_{k^{\prime}}(\varphi(t)) d s d t
$$

where $f_{k, k^{\prime}}, k, k^{\prime}=-N, \ldots, N$ are unknowns that need be to determined. Consequently, substituting the Sinc collocation points $x_{i}, y_{j}, i, j=-N, \ldots, N$ into equation (2.1) to 
have the system

$$
\begin{aligned}
& g\left(x_{i}, y_{j}\right)=\sum_{k=-N}^{N} \sum_{k^{\prime}=-N}^{N} f_{k, k^{\prime}}\left(\int_{a}^{x_{i}} \int_{a}^{y_{j}} k\left(x_{i}, y_{j}, s, t\right) S_{k}(\varphi(s)) S_{k^{\prime}}(\varphi(t)) d s d t\right) \\
& i, j=-N, \ldots, N
\end{aligned}
$$

The system (2.2) can be written in the matrix equation as follows;

$$
G=A F
$$

where

$$
G=\left[g\left(x_{-N}, y_{-N}\right), \mathrm{g}\left(x_{-N}, y_{-N+1}\right), \ldots, g\left(x_{-N}, y_{N}\right), g\left(x_{-N+1}, y_{-N}\right), \ldots, \mathrm{g}\left(x_{-N+1}, y_{N}\right), \ldots, g\left(x_{N}, y_{N}\right)\right]
$$

$F=\left[f\left(x_{-N}, y_{-N}\right), f\left(x_{-N}, y_{-N+1}\right), \ldots, f\left(x_{-N}, y_{N}\right), f\left(x_{-N+1}, y_{-N}\right), \ldots, f\left(x_{-N+1}, y_{N}\right), \ldots, f\left(x_{N}, y_{N}\right)\right]$

and

$$
A=\left[\begin{array}{ccccc}
A_{-N,-N}\left(x_{-N}, y_{-N}\right) & \ldots & A_{-N, N}\left(x_{-N}, y_{-N}\right) & A_{-N+1,-N}\left(x_{-N}, y_{-N}\right) & A_{-N+1, N}\left(x_{-N}, y_{-N}\right) \ldots A_{N, N}\left(x_{-N}, y_{-N}\right) \\
A_{-N,-N}\left(x_{-N+1}, y_{-N}\right) & \ldots & A_{-N, N}\left(x_{-N+1}, y_{N}\right) & A_{-N+!,-N}\left(x_{-N+1}, y_{-N}\right) & A_{-N+!, N}\left(x_{-N}, y_{-N}\right) \ldots A_{N, N}\left(x_{-N+1}, y_{-N}\right) \\
\cdot & \cdot & \cdot & \cdot & \cdot \\
\cdot & \cdot & \cdot & \cdot & \cdot \\
\cdot & \cdot & \cdot & \cdot & \cdot \\
A_{-N,-N}\left(x_{N}, y_{N}\right) & \ldots & A_{-N, N}\left(x_{N}, y_{N}\right) & A_{-N+!,-N}\left(x_{N}, y_{N}\right) & A_{-N+!, N}\left(x_{N}, y_{N}\right) \ldots A_{N, N}\left(x_{N}, y_{N}\right)
\end{array}\right]
$$

Such that $A_{i, j}(x, y)=\int_{a}^{x} \int_{a}^{y} k(x, y, s, t) S_{i}(\varphi(s)) S_{j}(\varphi(t)) d s d t, i, j=-N, \ldots, N$

By solving equation (2.3) using inverse method as $F=A^{-1} G$, then we will obtain the approximation solution for the unknown function $f(x, y)$. In fact, if the matrix $A$ is singular, then an approximation inverse could be evaluated by using the Pseudo inverse matrix.

Now, the same process could be used to solve equation (1.2) where the unknown function $f(x, y)$ of equation (1.2) is approximated by Sinc function interpolation (1.9) , substituting equation (1.9) into equation (1.2), then substituting the Sinc collocation points $x_{k}, y_{k^{\prime}}, k, k^{\prime}=-N, \ldots, N$ to have a linear system of the unknowns $f_{k, k^{\prime}}, k, k^{\prime}=-N, \ldots, N$ of the form 


$$
\begin{aligned}
& g\left(x_{i}, y_{j}\right)=\sum_{k=-N}^{N} \sum_{k^{\prime}=-N}^{N} f_{k, k^{\prime}}\left(S_{k}\left(\varphi\left(x_{i}\right)\right) S_{k^{\prime}}\left(\varphi\left(y_{j}\right)\right)-\int_{a}^{x_{i}} \int_{a}^{y_{j}} k\left(x_{i}, y_{j}, s, t\right) S_{k}(\varphi(s)) S_{k^{\prime}}(\varphi(t)) d s d t\right) \\
& i, j=-N, \ldots, N
\end{aligned}
$$

let

$$
B_{k, k^{\prime}}(x, y)=S_{k}(\varphi(x)) S_{k^{\prime}}(\varphi(y))-\int_{a}^{x} \int_{a}^{y} k(x, y, s, t) S_{k}(\varphi(s)) S_{k^{\prime}}(\varphi(t)) d s d t
$$

Then the system (2.7) can be written in matrix equation

$$
B G=F
$$

where. $B=\left[B_{i, j}\right]$.

\section{CONVERGE ANALYSIS}

We present an error bound for the approximate solution and we obtain a convergence order for the method. To this end, we consider the following theorem where the proof based on [10]

Theorem 1. If $f(x, y)$ is the exact solution of (1.1) and $k(x, y, s, t)$ continuous on $[a, b]^{2} \times[a, b]^{2}$, let

$$
P_{N}^{N}(f)(x, y)=\sum_{k=-N}^{N} \sum_{k^{\prime}=N}^{N} f_{k, k^{\prime}}^{N} S_{k}(\varphi(x)) S_{k^{\prime}}(\varphi(y))
$$

the Sinc function interpolation of $f(x, y)$, where $f_{k, k^{\prime}}^{N}, k, k^{\prime}=-N, \ldots, N$ are the coefficients that are determined by solving the matrix equation (2.3), then

$$
\left\|f(x, y)-P_{N}^{N}(f)\right\|_{\infty} \leq \beta N^{\frac{1}{2}} \exp -(\pi d \alpha N)^{\frac{1}{2}}
$$

where $\beta$ is constants independent of $N$.

Proof: let

$$
P_{N}(f(x, y))=\sum_{k=-N}^{N} \sum_{k^{\prime}=-N}^{N} f_{k, k^{\prime}} S_{k}(\varphi(x)) S_{k}(\varphi(y))
$$

Be the Sinc interpolation for the function $f(x, y)$ where $f_{k, k^{\prime}}=f\left(\varphi^{-1}(k h), \varphi^{-1}\left(k^{\prime} h\right)\right)$ the exact values of the function $f(x, y)$ at the Sinc collocation points $\left(x_{k}=\varphi^{-1}(k h), y_{k^{\prime}}=\varphi^{-1}\left(k^{\prime} h\right)\right)$. 
By substituting equations (3.1) and (3.2) into the integral equation (1.1) we have the following equations

$$
\begin{aligned}
& g(x, y)=\int_{a}^{x} \int_{a}^{y} k(x, y, s, t) P_{N}^{N}(f(s, t)) d s d t \\
& \dot{g}(x, y)=\int_{a}^{x} \int_{a}^{y} k(x, y, s, t) P_{N}(f(s, t)) d s d t
\end{aligned}
$$

To obtain the coefficients $f_{k, k^{\prime}}=f\left(\varphi^{-1}(k h), \varphi^{-1}\left(k^{\prime} h\right)\right)$ and $f_{k, k^{\prime}}^{N}$ of equations (3.2) and(3.1) respectively, we substitute the Sinc collocation points $\left(x_{k}, y_{k^{\prime}}\right)$ to have a linear system of equations of the unknowns $f_{k, k^{\prime}}$ that can be solved using inverse method as in previous section such that

$$
\left[f_{-N, N} \cdot \cdot f_{N, N}\right]=A^{-1}\left[\dot{g}\left(x_{i}, y_{j}\right)\right]_{i, j=-N}^{N}
$$

and

$$
\left[f_{-N, N}^{N} \cdot \cdot \cdot f_{N, N}^{V}\right]=A^{-1}\left[g\left(x_{i}, y_{j}\right)\right]_{i, j=-N}^{N}
$$

where $A$ is defined in equation(2.6). So that

$$
\sup _{k, k^{\prime} \in \operatorname{In}[-N, N]}\left|f_{k, k^{\prime}}^{N}-f_{k, k^{\prime}}\right| \leq\left\|A^{-1}\right\| \sup _{i, j \in \operatorname{In}[-N, N]}\left|g\left(x_{i}, y_{j}\right)-\dot{g}\left(x_{i}, y_{j}\right)\right|
$$

where $\operatorname{In}[-N, N]$ is the set of all integers in $[-N, N]$. Now subtracting equation (1.1) from equation (3.4), then we get

$$
\dot{g}(x, y)-g(x, y)=\int_{a}^{x} \int_{a}^{y} k(x, y, s, t)\left[f(s, t)-P_{N}(f(s, t))\right] d s d t
$$

Then

$$
\begin{aligned}
\sup _{i, j \in \operatorname{In}[-N, N]}\left|\dot{g}\left(x_{i}, y_{j}\right)-g\left(x_{i}, y_{j}\right)\right| & =\sup _{i, j \in \operatorname{In}[-N, N]}\left|\int_{a}^{x_{i}} \int_{a}^{y_{j}} k\left(x_{i}, y_{j}, s, t\right)\left[f(s, t)-P_{N}(f(s, t))\right] d s d t\right| \\
& \leq \int_{a}^{x_{i}} \int_{a}^{y_{j}} \sup _{\substack{i, j \in \operatorname{In}[-N, N] \\
s, t \in[-N, N]}}\left|k\left(x_{i}, y_{j}, s, t\right)\right|\left\|f(s, t)-P_{N}(f(s, t))\right\| d s d t \\
& \leq(b-a)^{2} M\left\|f-P_{N}\right\|, a<x_{i}, y_{j}<b, i, j=-N, \ldots, N
\end{aligned}
$$

where $M=\sup |k|$, by using theorem 2 and (3.6) equation (3.5) becomes as

$$
\sup _{k, k^{\prime} \in \operatorname{En}[-N, N]}\left|f_{k, k^{\prime}}^{N}-f_{k, k^{\prime}}\right| \leq\left\|A^{-1}\right\|(b-a)^{2} M\left(c_{1}+c_{2} \log N\right) N^{\frac{1}{2}} \exp -(\pi \alpha d N)^{\frac{1}{2}}
$$


Now

$$
\begin{aligned}
& \sup \left|\left(P_{N}^{N}-P_{N}\right)(f(x, y))\right|=\sup \left|\sum_{k=-N}^{N} \sum_{k^{\prime}=-N}^{N}\left(f_{k, k^{\prime}}^{N}-f_{k, k^{\prime}}\right) S_{k}(x) S_{k^{\prime}}(y)\right| \\
& \quad \leq\left\|A^{-1}\right\|(b-a)^{2} M\left(c_{1}+c_{2} \log (N)\right) N^{\frac{1}{2}} \exp -(\pi \alpha d N)^{\frac{1}{2}} \sup \left|\sum_{k=-N}^{N} \sum_{k^{\prime}=-N}^{N} S_{k}(x) S_{k^{\prime}}(y)\right| \\
& \quad \leq\left\|A^{-1}\right\|(b-a)^{2} M\left(c_{1}+c_{2} \log (N)\right) N^{\frac{1}{2}} \exp -(\pi \alpha d N)^{\frac{1}{2}} \frac{4}{\pi^{2}}(3+\log N)^{2}
\end{aligned}
$$

By using triangle inequality, we have

$$
\left\|f-P_{N}^{N}\right\| \leq\left(c_{2}+c_{3} \log N\right) N^{\frac{1}{2}} \exp -(\pi d \alpha N)^{\frac{1}{2}}\left(1+\frac{4}{\pi^{2}}(3+\log N)^{2}\right)=\beta \exp -(\pi d \alpha N)^{\frac{1}{2}} .
$$

The proof is completed.

Theorem: If $f(x, y)$ is the exact solution of (1.2) and $k(x, y, s, t)$ continuous on $[a, b]^{2} \times[a, b]^{2}$, let

$$
P_{N}^{N}(f)(x, y)=\sum_{k=-N}^{N} \sum_{k^{\prime}=N}^{N} f_{k, k^{\prime}}^{N} S_{k}(\varphi(x)) S_{k^{\prime}}(\varphi(y))
$$

Is the Sinc function interpolation of $f(x, y)$, where $f_{k, k^{\prime}}^{N}, k, k^{\prime}=-N, \ldots, N$ are the coefficients that are determined by solving the matrix equation (2.3), then

$$
\left\|f(x, y)-P_{N}^{N}(f)\right\|_{\infty} \leq\left(c_{2}+c_{3} \log N\right) N^{\frac{1}{2}} \exp -(\pi d \alpha N)^{\frac{1}{2}}\left(1+\frac{4}{\pi^{2}}(3+\log N)^{2}\right)
$$

where $c_{1}$ and $c_{2}$ are constants independent of $N$.

Proof: According to equations (3.1) and (3.2) which are the Sinc function approximation and Sinc interpolation of the function $f(x, y)$, then we consider the equations

$$
\begin{aligned}
& g(x, y)=P_{N}^{N}(f(x, y))-\int_{a}^{x} \int_{a}^{y} k(x, y, s, t) P_{N}^{N}(f(s, t)) d s d t \\
& \xi(x, y)=P_{N}(f(x, y))-\int_{a}^{x} \int_{a}^{y} k(x, y, s, t) P_{N}(f(s, t)) d s d t
\end{aligned}
$$

Where the coefficients $f_{k, k^{\prime}}$ and $f_{k, k^{\prime}}^{N}$ are obtained by substituting the Sinc collocation points into the above equations, then solving the linear systems as in previous theorem, then

$$
\sup _{k, k^{\prime} \in \operatorname{In}[-N, N]}\left|f_{k, k^{\prime}}^{N}-f_{k, k^{\prime}}\right| \leq\left\|B^{-1}\right\| \sup _{i, j \in \operatorname{In}[-N, N]}\left|g\left(x_{i}, y_{j}\right)-\xi\left(x_{i}, y_{j}\right)\right|
$$


By subtracting equation (1.2) from (3.11) to get

$$
\xi(x, y)-g(x, y)=f(x, y)-P_{N}(f(x, y)) \int_{a}^{x} \int_{a}^{y} k(x, y, s, t)\left[f(s, t)-P_{N}(f(s, t))\right] d s d t
$$

Then

$$
\begin{aligned}
\sup _{i, j \in I n[-N, N]}\left|\zeta\left(x_{i}, y_{j}\right)-g\left(x_{i}, y_{j}\right)\right|= & \sup _{i, j \in I n[-N, N] \mid}\left|\begin{array}{l}
\left(f\left(x_{i}, y_{j}\right)-P_{N}\left(f\left(x_{i}, y_{j}\right)\right)\right)- \\
\leq
\end{array} \int_{a}^{b} k\left(x_{i}, y_{j}, s, t\right)\left[f(s, t)-P_{N}(f(s, t))\right] d s d t\right| \\
\leq & \left\|f(s, t)-P_{N}(f(s, t))\right\|+ \\
& \int_{a}^{b} \int_{a}^{b} \sup _{\substack{i, j \in I n[-N, N] \\
s, t \in[-N, N]}} \mid k\left(x_{i}, y_{j}, s, t\right)\left\|f(s, t)-P_{N}(f(s, t))\right\| d s d t \\
\leq & \left((b-a)^{2} M+1\right)\left\|f-P_{N}\right\| \\
\leq & \left((b-a)^{2} M+1\right)\left(c_{1}+c_{2} \log N\right) N^{\frac{1}{2}} \exp \left(-(\pi \alpha d N)^{\frac{1}{2}}\right)
\end{aligned}
$$

so

$$
\sup _{k, k^{\prime} \in \operatorname{In}[-N, N]}\left|f_{k, k^{\prime}}^{N}-f_{k, k^{\prime}}\right| \leq\left\|B^{-1}\right\|\left((b-a)^{2} M+1\right)\left(c_{1}+c_{2} \log N\right) N^{\frac{1}{2}} \exp -(\pi \alpha d N)^{\frac{1}{2}}
$$

and

$$
\begin{aligned}
& \sup \left|\left(P_{N}^{N}-P_{N}\right)(f(x, y))\right|=\sup \left|\sum_{k=-N}^{N} \sum_{k^{\prime}=-N}^{N}\left(f_{k, k^{\prime}}^{N}-f_{k, k^{\prime}}\right) S_{k}(x) S_{k^{\prime}}(y)\right| \\
& \leq\left\|B^{-1}\right\|\left((b-a)^{2} M+1\right)\left(c_{1}+c_{2} \log (N)\right) N^{\frac{1}{2}} \exp -(\pi \alpha d N)^{\frac{1}{2}} \frac{4}{\pi^{2}}(3+\log N)^{2}
\end{aligned}
$$

Finally,

$$
\begin{aligned}
& \left\|f-P_{N}^{N}(f)\right\| \leq\left\|f-P_{N}(f)\right\|+\left\|P_{N}(f)-P_{N}^{N}(f)\right\| \\
& \leq\left(\left\|B^{-1}\right\|\left((b-a)^{2} M+1\right) \frac{4}{\pi^{2}}(3+\log N)^{2}+1\right)\left(c_{1}+c_{2} \log (N)\right) N^{\frac{1}{2}} \exp -(\pi \alpha d N)^{\frac{1}{2}} \\
& =\text { constant } \exp -(\pi \alpha d N)^{\frac{1}{2}} .
\end{aligned}
$$

\section{NUMERICAL EXAMPLES}

In this section, we give some numerical examples to clarify the efficiency of the presented method. We will consider two-dimensional Volterra integral equations of 
first and second kind. The examples have been solved with $\alpha=1, d=\frac{\pi}{2}$ and different values of $N$.

Example 1. For equation (1.1), let $g(x, y)=\frac{x^{3} y^{3}}{9} e^{x+y}, k(x, y, s, t)=e^{x+y} s t$, the exact solution $f(x, y)=x y, x, y \in(0,1)$ and $\quad$ let $\quad\left|E_{N}\right|=\max _{-N \leq x_{k}, y_{k^{\prime}} \leq N}\left|f\left(x_{k}, y_{k^{\prime}}\right)-P^{N}\left(x_{k}, y_{k^{\prime}}\right)\right|$, where $x_{k}, y_{k^{\prime}}$ are the Sinc collocation points. Table 1 shows the values for the error with different values of $N$.

Example 2. For equation (1.2), let $g(x, y)=\cos x \sin y-\cos x y(x \sin x+\cos x-1)(\sin y-y \cos y)$, and $k(x, y, s, t)=s t \cos x y$, and the exact solution $f(x, y)=\cos x \sin y, x, y \in[0,1]$. Let $\left|E_{N}\right|=\max _{-N \leq x_{k}, y_{k^{\prime}} \leq N}\left|f\left(x_{k}, y_{k^{\prime}}\right)-P^{N}\left(x_{k}, y_{k^{\prime}}\right)\right|$, the numerical results are given in table 2.

Table 1. Results for example 1 and 2.

\begin{tabular}{|l|l|l|l|l|l|}
\cline { 2 - 6 } \multicolumn{1}{c|}{} & $N$ & 5 & 10 & 20 & 30 \\
\hline Example1 & $\left|E_{N}\right|$ & $5.5602 \times 10^{-3}$ & $6.8195 \times 10^{-4}$ & $9.4701 \times 10^{-8}$ & $2.5481 \times 10^{-9}$ \\
\hline Example 2 & $\left|E_{N}\right|$ & $9.7345 \times 10^{-2}$ & $1.4328 \times 10^{-3}$ & $5.7834 \times 10^{-6}$ & $6.1295 \times 10^{-8}$ \\
\hline
\end{tabular}

\section{CONCLUSION}

In this paper, we designed a simple high accurate method for solving two-dimensional Volterra integral equations of the first kind and second by using Sinc collocation method. Moreover, we proved of the method, and given examples applied for first and second kind of integral equations show, the presented method has high accuracy. Also, it will be possible to investigate the numerical solution of the nonlinear twodimensional Volterra integral equations of first and second kind, and the twodimensional integral-algebraic equations of different indexes.

\section{REFERENCES}

[1] Mohammed Heydari and Zakieh Avazzadeh, "Chebyshev polynomials for solving two dimensional linear and nonlinear integral equations of the second kind," Computational \& Applied Mathematics, vol. 31, no. 1, pp. 127-142, 2012.

[2] B.A. Beltyukov and L.N. Kuznechikhina,, "A Runge \pm Kutta method for the solution of twodimensional," Differential Equations, vol. 12, pp. 1169-1173, 
1976.

[3] Han Guoqiang, Ken Hayami, Kokichi Sugihara and Wang Jiong, "Extrapolation method of iterated collocation solution for two-dimensional nonlinear Volterra integral equations," Applied Mathematics and Computation, vol. 112, p. 49 \pm 61 , 2000 .

[4] R.Katani and S. Shahmorad, "A New Block by Block Method for Solving TwoDimensional Linear and Linear Volterra Integral Equations of the First and Second Kind," Bulletin of the Iranian Mathematical Society, vol. 39, no. 4, pp. 707-724, 2013.

[5] K. Maleknejad, S. Sohrabi and B. Baranji, "Two-Dimensional PCBFs: Applications to Nonlinear Volterra Integral Equations," in World Congress on Engineering, London, 2009.

[6] E. Babolian and N. Dastani, "Numerical Solutions of Two-dimensional Linear and Nonlinear Volterra Integral Equations:Homotopy Perturbation Method and Differential Transform Method," International Journal of Industrial Mathematics, vol. 3, no. 3, pp. 157-167, 2011.

[7] A. TariI and S. Shahmorad, "Numerical Solution of A Class of Two-Dimensional Nonlinear Volterra Integral Equations of the First Kind," Journal of Applied Mathematics \& Infornatics, vol. 30, no. 3-4, pp. 463-475, 2012.

[8] M. I. Berengue and D .G'amez, "A computational method for solving a class of two dimensional," Journal of Computational and Applied mathematics, vol. Volume 318, p. 403-410, 2017.

[9] Guqiang Han and Ruifang Wangn, "The Extrapolation Method for TwoDimensional Volterra Integral Equations Based on the Asmptotic Expansion of Iterated Galerkin Solutions," Journal of Integral Equations and Applications, vol. 13, no. 1, pp. 15-35, 2001.

[10] Mirzaee Farshid, "Solving a Class of Nonlinear Two-Dimensional Volterra Integral Equations by Uing Two-Dimensional Triangular Orthogonal Functions," Journal Of Mathematical Modeling, vol. 1, no. 1, pp. 28-40, 2013.

[11] Morteza Khodabin, Khosrow Maleknejad and Mohsen Fallahpour, "Approximation Solution of Two-Dimensional Stochastic Fredholm Integral Equation by Applying the Haar Wavelet," International Journal of Mathematical Modeling \$ Computations, vol. 5, no. 4, pp. 1-17, 2015.

[12] Yousef Aljarrah and En-Bing Lin, "Wavelet Based Methods for Numerical Solutions of Two Dimensional Integral Equations," Mathematica Aeterna, vol. 4, no. 8 , pp. 839-853, 2014.

[13] En-Bing Lin and Yousef Al-Jarrah , "Numerical Solution of Freholm-Volterra Integral Equations by Using Scaling Function Interpolation Method," Applied 
Mathematics, vol. 4, pp. 204-209, 2013.

[14] Yousef Al-Jarrah and En-Bing Lin, "Wavelet Interpolation Method for Solving Singular Integral Equations," Applied Mathematics, vol. 4, pp. 1-4, 2013.

[15] M. Zarebnia, "Convergence of the Sinc method applied," Applications and Applied, vol. 5, no. 1, pp. 198-216, 2010.

[16] Khosrow Maleknejad, Reza Mollapourasl,Parvin Torabi and Mahdiyeh Alizadeh, "Solution of First kind Fredholm Integral Equation," International Journal of Mathematical, Computational, Physical, Electrical and Computer Engineering, vol. 4, no. 6, pp. 737-741, 2010.

[17] Stenger Frank, Numerical Methods Based on Sinc and Analytic Functions, Salt Lake City, USA: Springer, 1993. 